\title{
Thank you to the reviewers
}

\author{
J. W. F. van Mil
}

Received: 28 November 2010/ Accepted: 6 December 2010/Published online: 13 January 2011

(C) Springer Science+Business Media B.V. 2010

The editorial staff of the International Journal of Clinical Pharmacy (IJCP, previously called Pharmacy World \& Science), wishes to thank the following reviewers, who have helped to maintain and improve the quality of the journal in 2010.

DerarAbdel-Qader, United Kingdom

Ahmend Zamzam Mohamed Ismail, United Kingdom

Gazala Akram, United Kingdom

Angela Alexander, United Kingdom

Anna Birna Almarsdottir, Iceland

Elske Ammenwerth, Austria

Claire Anderson, United Kingdom

Carol Armour, Australia

Parisa Aslani, Australia

Zaheer Ud din Babar, New Zealand

Douglas Ball, Netherlands

Peri Ballantyne, Canada

Roberto Banfi, Italy

Pierrick Bedouch, France

J. Simon Bell, Finland

Shalom Benrimoj, Australia

Cecilia Bernsten, Sweden

Yvonnick Bezie, France

Debi Bhattacharya, United Kingdom

Ingunn Bjornsdottir Iceland

Hege Blix, Norway

Lyda Blom, Netherlands

Helen Boardman, United Kingdom

This is an editorial contribution for the first issue of the International Journal of Clinical Pharmacy.

J. W. F. van Mil ( $\square)$

Margrietlaan 1, 9471 CT Zuidlaren, The Netherlands

e-mail: jwfvmil@planet.nl
Hendrikus Boersma, Netherlands

Christine Bond, United Kingdom

Sinthia Bosnic-Anticevich, Australia

Ian Boyd, Australia

Matthew Boyd, United Kingdom

Rhiannon Braund, New Zealand

Jo-Anne Brien, Australia

Koos Brouwers, Netherlands

David Brown, United Kingdom

José Cabrita, Portugal

Phillip Capps, Kuwait

Barry Carter, United States

Staffan Castensson, Sweden

Afonso Cavaco, Portugal

Li-Chia Chen, United Kingdom

Timothy Chen, Australia

Henry Chrystyn, United Kingdom

William Cottrell, Australia

Carolyn Coulter, New Zealand

Anthony Cox, United Kingdom

Anton de Boer, Netherlands

Han de Gier, Netherlands

Petra Denig, Netherlands

Franciska Desplenter, Belgium

Ermindo Di Paolo, Switzerland

Parastou Donyai, United Kingdom

R.L. Doty, United States

Gregory Duncan, Australia

Martin Eden, United Kingdom

Anders Ekedahl, Sweden

Lynne Emmerton, Australia

Dita Engová, Czech Republic

Anne Epema, Netherlands

Karen Farris, United States

Fernando Fernandez-Llimos, Portugal 
Thomas Fiss, Germany

Roel Fijn, Netherlands

Romano Fois, Australia

Jan Gärtner, Germany

Wilma Göttgens, Netherlands

Thijs Giezen, Netherlands

Dick Gourley, United States

G.G. Graham, Australia

Anne Granas, Norway

Gary Grant, Australia

Birgit Grave, Germany

Andrew Gray, South Africa

James Green, New Zealand

Nina Griese, Germany

Steven Grunberg, United States

Helle Hakonsen, Norway

Cyrine Haidar, United States

Devina Halsall, United Kingdom

Yolande Hanssens, Qatar

Jeffrey Harrison, New Zealand

Georg Hempel, Germany

Martin Henman, Ireland

Peter Herbison, New Zealand

Hanne Herborg, Denmark

Kurt Hersberger, Switzerland

Simon Horsburgh, New Zealand

Carmel Hughes, United Kingdom

Jacqueline Hugtenburg, Netherlands

M. Izham Ibrahim, Saudi Arabia

Jenni Ilomaki, Finland

Ulrich Jaehde, Germany

Delyth James, United Kingdom

Shazia Jamshed, Malaysia

Katrin Janhsen, Germany

Chris Jay, New Zealand

Therese Kairuz, Australia

Frieder Keller Germany

Nadir Kheir, Qatar

Michelle King, Australia

Moira Kinnear, United Kingdom

Maarit Korhonen, Finland

Mitchell Kostich, United States

Stephan Krahenbuhl, Switzerland

Ines Krass, Australia

Hannelore Kreckel, Germany

Janet Krska, United Kingdom

Stephanie Läer, Germany

Raisa Laaksonen, Finland

Penny Lewis, United Kingdom

Sunny Linnebur, United States

Christina Ljungberg, Sweden

Judith Lowthian, Australia

Christine Lu, Australia
Ann-Charlotte Mardby, Sweden

Adam Mackridge, United Kingdom

Anke Hilse Maitland-van der Zee, Netherlands

Louise Mallet, Canada

Eduardo Mariño, Spain

Jan Gerard Maring, Netherlands

Linda Gore Martin, United States

Pamela Mason, United Kingdom

Wandikayi Matowe, Qatar

Patrick McDonnell, United States

R.P. McDonough, United States

James C. McElnay, United Kingdom

Lorna McHattie, United Kingdom

Cathy McKenzie, United Kingdom

Saran McLoughlin-Braybrook, United Kingdom

Els Mehuys, Belgium

Jurian Meijering, Australia

Andrea Messori, Italy

Jan Meulenbelt, Netherlands

Andrew Miesner, United States

Mark P. Mobach, Netherlands

Klaus Moerike, Germany

Claus Moldrup, Denmark

Rebekah Moles, Australia

Charles Morecroft, United Kingdom

Janine Morgall Traulsen, Denmark

Hans Mulder, Netherlands

Kirsten Myhr, Norway

Stephanie Natsch, Netherlands

Cees Neef, Netherlands

Lars Nilsson, Sweden

Hedvig Nordeng, Norway

Pauline Norris, New Zealand

Carole Parsons, United Kingdom

Denham Phipps, United Kingdom

Marika Pohjanoksa-Mantyla, Finland

Lisa Pont, Australia

Terry Porteous, United Kingdom

Maarten Postma, Netherlands

Yasmine Probst, Australia

Roland Radziwill, Germany

Jean-Baptiste Rey, France

Nate Rickles, United States

André Rieutord France

Maria Rikala, Finland

Hugo Robays, Belgium

Colin Rousseaux, Canada

Cristin Ryan, United Kingdom

Birthe Sondergaard, Denmark

Ellen Sorensen, Denmark

Laura Sahm, Ireland

Bandana Saini, Australia

Imogen Savage, United Kingdom 
Nicolas Schaad, Switzerland Marion Schaefer, Germany Ellen Schafheutle, United Kingdom Tom Schalekamp, Netherlands Martin Schulz, Germany Janie Sheridan, New Zealand Olayinka Shiyanbola, United States Robert Shulman, United Kingdom Sofia Kalvermark, Sporrong Sweden Rebecca Stack, United Kingdom Derek Stewart, United Kingdom Waleed Sweileh, Palestine Klara Tisocki, Kuwait

Hilde Tobi, Netherlands

Daan Touw, Netherlands

Sabrina Trippoli, Italy Mary Tully, United Kingdom Patricia van den Bemt, Netherlands Karen van Dijk, Netherlands
E.M. van Maarseveen, Netherlands Alfons Verbruggen, Belgium Charlotte Verrue, Belgium Pierre Voirol, Switzerland R. Voskuyl, Netherlands Geoffrey Wall, United States Warangkana Warisnoicharoen, Thailand Margaret Watson United Kingdom Albert Wertheimer, United States Tone Westergren, Norway Tommy Westerlund, Sweden Jasmin Widmann, United Kingdom Kerry Wilbur, Qatar Bob Wilffert, Netherlands Kylie Williams, Australia Joy Wingfield, Australia Almut Winterstein, United States R.J. Ziance, United States 